PROCEEDINGS OF THE

AMERICAN MATHEMATICAL SOCIETY

Volume 129, Number 3, Pages 753-758

S 0002-9939(00)05588-X

Article electronically published on September 19, 2000

\title{
NEARLY CONVEX FUNCTIONS, PERTURBATIONS OF NORMS AND $K$-SPACES
}

\author{
FÉLIX CABELLO SÁNCHEZ
}

(Communicated by Dale Alspach)

\begin{abstract}
We prove several results connecting the stability of convex maps (defined on the unit ball of a Banach space) to the notion of $K$-space.
\end{abstract}

\section{INTRODUCTION}

This paper deals with the "Hyers-Ulam" problem of finding conditions under which an approximately subadditive (or convex) function must be near to a true subadditive (or convex) function. As far as we know, approximately convex maps were first studied by Hyers and Ulam [HU]. They considered $\varepsilon$-convex functions, that is, functions satisfying

$$
f(h x+(1-h) y) \leq h f(x)+(1-h) f(y)+\varepsilon
$$

$(0 \leq h \leq 1)$ and proved that to each $\varepsilon$-convex function defined on a convex subset $S$ of an $n$-dimensional space there corresponds a convex $g$ such that $|f(x)-g(x)| \leq$ $K_{n} \varepsilon$, where $K_{n}$ is a constant depending only on $n$. The best known estimate is $K_{n} \leq\left(1+\left[\log _{2} n\right]\right) / 2$; see Ch, section 2].

Later Casini and Papini [CP] gave a nice example showing that in the infinite dimensional case the stability theorem of [HU] does not hold. For a suitable convex bounded subset $S$ of $l_{1}$, they construct a parametric family of maps

$$
f_{p}: S \subset l_{1} \rightarrow \mathbb{R}
$$

$(0<p<1)$ such that $f_{p}$ is $\left(2^{1 / p}-2\right)$-convex and there is no $K$ such that for each $p$

$$
\left|f_{p}(x)-g(x)\right| \leq K\left(2^{1 / p}-2\right)
$$

holds for some convex $g$. Nevertheless, for each $p$ there is a convex $g$ such that $\left|f_{p}(x)-g(x)\right|$ is bounded on $S$ since every $f_{p}$ has Lipschitz constant 1. (We shall see that there exist much more extreme counterexamples.)

As is remarked in $[\mathrm{CP}$, the stability problem as stated is in some sense independent of the norm-topology of the underlying Banach space. This is due to the fact that the measurement of the degree of convexity of a given function is unrelated to the metric of the space and therefore the behavior of approximately convex maps can say almost nothing about the Banach space on which they are defined.

Received by the editors April 14, 1998 and, in revised form, May 5, 1999.

2000 Mathematics Subject Classification. Primary 39B82, 39 B62.

The author was supported in part by DGICYT project PB97-0377 and HI project 1997-0016. 
A way to avoid this problem is to consider functions defined on the unit ball of a given Banach space (a set which reflects the metric structure of the space).

We prove that $X$ is a $K$-space if and only if every homogeneous $\varepsilon$-convex map $B(X)$ (the unit ball of $X$ ) can be uniformly approximated by a convex one. Recall that a quasi-Banach space $X$ is said to be a $K$-space [KPR p. 82] if whenever $Z$ is a quasi-Banach space containing a subspace $L$ of dimension one with $Z / L$ isomorphic to $X$, then $L$ is complemented in $X$. By a result of Dierolf [Di], a Banach space $X$ is a $K$-space if and only if whenever

$$
0 \rightarrow Y \rightarrow Z \rightarrow X \rightarrow 0
$$

is an exact sequence of quasi-Banach spaces and operators in which $Y$ is locally convex the middle space $Z$ is also locally convex. For instance, $B$-convex spaces (i.e., Banach spaces having non-trivial type) and quotients of $C(K)$ spaces are $K$-spaces ( $\mathrm{Ka}],[\mathrm{KR}]$ ) while $l_{1}$ is not $([\mathrm{Ri})$.

Thus if $X$ fails to be a $K$-space (for instance, if $X$ contains $l_{1}^{n}$ uniformly complemented), then for every $\varepsilon>0$ there exists an $\varepsilon$-convex map on $B(X)$ that cannot be uniformly approximated by any convex map. We give a simple example for $X=l_{1}$, whose finite dimensional versions show that, for the constants of Hyers and Ulam, one has $K_{n} \geq 1 / 2 \log _{2} n$, improving the results of $[\mathrm{CP}$. This also shows that the estimate of Cholewa $K_{n} \leq\left(1+\left[\log _{2} n\right]\right) / 2$ quoted above is almost sharp.

As a complement, we prove that, to some extent, the stability of the triangle inequality is a characteristic property of $K$-spaces: $X$ is a $K$-space if and only if for every absolutely homogeneous map $f: X \rightarrow \mathbb{R}(f(t x)=|t| f(x))$ which is "nearly convex" in the sense that

$$
f(x+y) \leq f(x)+f(y)+\varepsilon\{\|x\|+\|y\|\},
$$

the "perturbed functional" $x \in X \rightarrow\|x\|+f(x)$ is equivalent to a (convex) norm on $X$ (which may be not equivalent to the original norm of $X$ ).

\section{Nearly CONVEX functions and $K$-SPaCes}

Recall that a mapping $f: X \rightarrow \mathbb{R}$ is called quasi-linear if it is homogeneous and quasi-additive in the sense that

$$
|f(x+y)-f(x)-f(y)| \leq \varepsilon\{\|x\|+\|y\|\}
$$

holds for some $\varepsilon$ and all $x, y$ in $X$. One has the following fundamental result (see [KPR, p. 90, Corollary 5.6] or [Ka p. 257, Proposition 3.3]).

Theorem 1. A Banach space $X$ is a $K$-space if and only if to every quasi-linear map $f: X \rightarrow \mathbb{R}$ there corresponds a linear (not necessarily bounded) functional $L$ such that $|f(x)-L(x)| \leq K\|x\|$ for some $K$ and all $x$.

We are ready to prove the following

Theorem 2. A Banach space $X$ is a $K$-space if and only if every $\varepsilon$-convex homogeneous map on $B(X)$ can be uniformly approximated by a convex one.

Proof. The "only if" part is clear. For the converse, let $f: X \rightarrow \mathbb{R}$ be quasilinear with constant $\varepsilon$. Then the restriction of $f$ on $B(X)$ is $\varepsilon$-convex since for $x$, 
$y \in B(X)$ and $0 \leq h \leq 1$ one has

$$
\begin{aligned}
f(h x+(1-h) y) & \leq f(h x)+f((1-h) y)+\varepsilon\{\|h x\|+\|(1-h) y\|\} \\
& =h f(x)+(1-h) f(y)+\varepsilon\{|h|\|x\|+|1-h|\|y\|\} \\
& \leq h f(x)+(1-h) f(y)+\varepsilon .
\end{aligned}
$$

The hypothesis gives a convex $g$ such that $|f(x)-g(x)| \leq K$ for some $K$ and all $x$ in $B(X)$. It follows that for any convex combination of points of the unit ball one has

$$
f\left(\sum_{i=1}^{n} h_{i} x_{i}\right) \leq \sum_{i=1}^{n} h_{i} f\left(x_{i}\right)+K
$$

Using homogeneity one obtains the following property of $f$ : if $\left\{x_{i}\right\}$ is a finite subset of $X$, then

$$
f\left(\sum_{i=1}^{n} x_{i}\right) \leq \sum_{i=1}^{n} f\left(x_{i}\right)+K n\left\{\max _{1 \leq i \leq n}\left\|x_{i}\right\|\right\}
$$

which implies that, in fact,

$$
f\left(\sum_{i=1}^{n} x_{i}\right) \leq \sum_{i=1}^{n} f\left(x_{i}\right)+K\left\{\sum_{i=1}^{n}\left\|x_{i}\right\|\right\} .
$$

Put $p(x)=\inf \left\{\sum_{i=1}^{n} f\left(x_{i}\right)+K \sum_{i=1}^{n}\left\|x_{i}\right\|: x=\sum_{i} x_{i}\right\}$. Then $p$ is subadditive and satisfies that $p(t x)=t p(x)$ for every $x$ in $X$ and all $t \geq 0$. The Hahn-Banach theorem gives a linear functional $L$ dominated by $p$. Thus, $L(x) \leq p(x) \leq f(x)+$ $K\|x\|$, from which it follows that

$$
L(x)-f(x) \leq K\|x\|
$$

Taking into account that both $L$ and $f$ are odd maps, one arrives at

$$
|f(x)-L(x)| \leq K\|x\|,
$$

which completes the proof.

Example 3. For each $\varepsilon>0$ there is an $\varepsilon$-convex homogeneous map on the unit ball of $l_{1}$ which cannot be uniformly approximated by any convex map. A concrete example is given by the Ribe map [Ri] as follows. Consider the map $f: \mathbb{R} \rightarrow \mathbb{R}$ given by $f(t)=t \log |t|$ (assuming that $f(0)=0$ ). It is not hard to see that $f$ is quasi-additive. In fact,

$$
|f(x+y)-f(x)-f(y)| \leq \log (2)\{|x|+|y|\} .
$$

Now define a map on the finitely supported sequences of $l_{1}$ by

$$
F(x)=\left(\sum_{i} x_{i}\right) \log \left|\sum_{i} x_{i}\right|-\sum_{i} x_{i} \log \left|x_{i}\right| .
$$

Clearly, $F$ is homogeneous. Moreover,

$$
|F(x+y)-F(x)-F(y)| \leq 2 \log (2)\{\|x\|+\|y\|\}
$$

so that $F$ is quasi-linear. Using known techniques $\left[\mathrm{KR}\right.$ it can be extended to $l_{1}$ keeping quasi-linearity. Therefore the extension is also $\varepsilon$-convex on the unit ball for some $\varepsilon$. Let us estimate the distance between the restriction of $F$ to $B_{n}$ (the unit ball of the subspace spanned by the first $n$ vectors of the usual basis of $l_{1}$ ) and an 
arbitrary convex map. Let $g: B_{n} \rightarrow \mathbb{R}$ be a convex map such that $|F(x)-g(x)| \leq d_{n}$ on $B_{n}$. Then

$$
\begin{aligned}
d_{n} & \geq F\left(\sum_{i=1}^{n} \frac{1}{n} e_{i}\right)-g\left(\sum_{i=1}^{n} \frac{1}{n} e_{i}\right) \\
& \geq \frac{1}{n}\left[f\left(\sum_{i=1}^{n} e_{i}\right)-\sum_{i=1}^{n} g\left(e_{i}\right)\right] \geq \frac{1}{n} F\left(\sum_{i=1}^{n} e_{i}\right)-d_{n},
\end{aligned}
$$

which yields $d_{n} \geq \log (n) / 2=\log (2) \log _{2}(n) / 2$. This shows that $F$ cannot be uniformly approximated by any convex map on the unit ball of $l_{1}$ and also that $K_{n} \geq \log _{2}(n) / 4$.

Remark. Actually, tedious computations show that $F$ is even $\log (2)$-convex when restricted to $B_{n} \cap\{x \geq 0\}$. This implies that $K_{n} \geq 1 / 2 \log _{2}(n)$, improving (and not only asymptotically!) the result in $[\mathrm{CP}]$.

\section{Perturbation of nORMs}

As a complement, we present the following theorem asserting that, in some sense, the stability of the triangle inequality is a characteristic property of $K$-spaces.

Theorem 4. A Banach space $X$ is a $K$-space if and only if for every absolutely homogeneous (non-negative) mapping $f: X \rightarrow \mathbb{R}$ satisfying

$$
f(x+y) \leq f(x)+f(y)+\varepsilon\{\|x\|+\|y\|\}
$$

the functional $x \in X \rightarrow\|x\|+f(x)$ is equivalent to a norm on $X$ (which may not be equivalent to the original norm of $X)$.

Proof. We first show the "only if" part (which surprisingly does not use quasilinear maps). Consider the functional $x \rightarrow\|x\|+f(x)$ defined on $X$. Clearly it is absolutely homogeneous. Moreover,

$$
\begin{aligned}
f(x+y)+\|x+y\| & \leq f(x)+f(y)+\varepsilon\{\|x\|+\|y\|\}+\|x+y\| \\
& \leq(1+\varepsilon)(f(x)+\|x\|+f(y)+\|y\|),
\end{aligned}
$$

so that $f+\|\cdot\|$ is a quasi-norm and $(X, f+\|\cdot\|)$ is a quasi-normed space. Obviously the formal identity $T:(X, f+\|\cdot\|) \rightarrow X$ is bijective and continuous. The space $(X, f+\|\cdot\|)$ may not be complete: it is complete if and only if $\|\cdot\|$ dominates $f$, by the Open Mapping Theorem. Observe that $\left(x_{n}\right)$ is a Cauchy sequence in $(X, f+\|\cdot\|)$ if and only if

$$
\lim _{n, m \rightarrow \infty}\left\|x_{n}-x_{m}\right\|=\lim _{n, m \rightarrow \infty} f\left(x_{n}-x_{m}\right)=0 .
$$

Thus, a Cauchy sequence in $(X, f+\|\cdot\|)$ is a convergent sequence in $X$ which is also a Cauchy sequence with respect to $f$. Let $Z$ be the completion of $(X, f+\|\cdot\|)$ (which always exists as a quasi-Banach space). Then $T$ extend to a continuous operator $Z \rightarrow X$ (denoted also by $T$ ) so that there is an exact sequence

$$
0 \rightarrow \operatorname{ker} T \rightarrow Z \rightarrow X \rightarrow 0 .
$$

We want to see that $\operatorname{ker} T$ is a Banach space (and not merely a quasi-Banach space). One needs a description of how $T$ acts on $Z$. Take $x^{*}$ in $Z$. Then $x^{*}=\left[\left(x_{n}\right)\right]$, where 
$\left(x_{n}\right)$ is convergent in $X$ and $\left(x_{n}\right)$ is a Cauchy sequence with respect to $f$. It follows that

$$
T\left(x^{*}\right)=\|\cdot\|-\lim _{n} x_{n} .
$$

So, elements of ker $T$ are null sequences in $X$ which are Cauchy sequences for $f$. We now prove that if $x^{*}=\left[\left(x_{n}\right)\right]$ belongs to $\operatorname{ker} T$, then $\lim _{n} f\left(x_{n}\right)$ exists (this is not obvious at all, since $f$ is not generally subadditive). From

$$
f(x+y) \leq f(x)+f(y)+\varepsilon\{\|x\|+\|y\|\},
$$

taking $x_{n}=x+y, x_{m}=y$, we get

$$
f\left(x_{n}\right) \leq f\left(x_{n}-x_{m}\right)+f\left(x_{m}\right)+\varepsilon\left\{\left\|x_{n}-x_{m}\right\|+\left\|x_{n}\right\|\right\},
$$

hence $f\left(x_{n}\right) \leq f\left(x_{m}\right)+\delta$ for $n$ and $m$ large enough. By symmetry we obtain that $\left|f\left(x_{n}\right)-f\left(x_{m}\right)\right| \rightarrow 0$ as $n, m \rightarrow \infty$.

Therefore the topology of ker $T$ comes from the (absolutely homogeneous) functional

$$
f^{*}\left(x^{*}\right)=\lim _{n} f\left(x_{n}\right),
$$

where $x^{*}=\left[\left(x_{n}\right)\right]$. But $f^{*}$ is actually a norm, since for $x^{*}=\left[\left(x_{n}\right)\right], y^{*}=\left[\left(y_{n}\right)\right]$ one has

$$
\begin{aligned}
f^{*}\left(x^{*}+y^{*}\right) & =\lim _{n} f\left(x_{n}+y_{n}\right) \\
& \leq \lim _{n} f\left(x_{n}\right)+\lim _{n} f\left(y_{n}\right)+\varepsilon\left\{\left\|x_{n}\right\|+\left\|y_{n}\right\|\right\} \\
& =\lim _{n} f\left(x_{n}\right)+\lim _{n} f\left(y_{n}\right) \\
& =f^{*}\left(x^{*}\right)+f^{*}\left(y^{*}\right) .
\end{aligned}
$$

Thus, in the exact sequence

$$
0 \rightarrow \operatorname{ker} T \rightarrow Z \rightarrow X \rightarrow 0,
$$

the left space is a Banach space. The hypothesis about $X$ implies that $Z$ is also isomorphic to a Banach space and there is a (convex) norm giving the topology of $Z$. Clearly, its restriction on $X$ is norm equivalent to $f+\|\cdot\|$ on $X$, as desired.

For the converse, let $f: X \rightarrow \mathbb{R}$ be quasi-linear with constant $\varepsilon$. Consider the twisted sum $\mathbb{R} \oplus_{f} X$ induced by $f$, that is, the product space $\mathbb{R} \times Y$ endowed with the quasi-norm

$$
\|(t, x)\|_{f}=|t-f(x)|+\|x\| .
$$

On the (generally non-closed) hyperplane $H=0 \times X$ the quasi-norm becomes

$$
\|(0, x)\|_{f}=|f(x)|+\|x\| \text {. }
$$

Since $x \rightarrow|f(x)|$ is absolutely homogeneous and satisfies that

$$
|f(x+y)| \leq|f(x)|+|f(y)|+\varepsilon\{\|x\|+\|y\|\},
$$

the hypothesis on $X$ implies that $H$ is a locally convex space. Now, if $H$ is closed in $\mathbb{R} \oplus_{f} X$, then $|f(\cdot)|+\|\cdot\|$ is equivalent to the original norm of $X$ and $f$ is bounded (in the sense that $|f(x)| \leq K\|x\|$ for some $K$ and all $x \in X$ ) and there is nothing to prove. Otherwise $H$ is dense in $\mathbb{R} \oplus_{f} X$ and therefore $\mathbb{R} \oplus_{f} X$ is itself locally convex. The rest is a standard deduction: the Hahn-Banach theorem guarantees the existence of a bounded linear functional $A: \mathbb{R} \oplus_{f} X \rightarrow \mathbb{R}$ such that $A(t, 0)=t$ 
for all $t$. Obviously this map can be written as $A(t, x)=t-L(x)$, with $L: X \rightarrow \mathbb{R}$ a linear (although not necessarily bounded) map. Nevertheless $A$ is bounded, so

$$
|t-L x|=|A(t, x)| \leq M\|(t, x)\|_{f}=M(|t-f(x)|+\|x\|) .
$$

Taking $t=f(x)$ one obtains that $|f(x)-L x| \leq M\|x\|$, which completes the proof.

Problem. Is it true that every $\varepsilon$-convex map defined on the unit ball of a $K$-space can be (uniformly) approximated by a convex one?

\section{REFERENCES}

[CP] E. Casini and P. L. Papini, A counterexample for the infinity version of the Hyers and Ulam stability Theorem, Proc. Amer. Math. Soc. 118 (1993), 885-890. MR 93i:26022

[Ch] P. W. Cholewa, Remarks on the stability of functional equations, Aequationes Math. 27 (1984), 76-86. MR 86d:39016

[Di] S. Dierolf, Über Vererbbarketiseigenschaften in topologischen Vektorräumen, Dissertation, Ludwig-Maximilian Univ., Munich, 1973.

[HU] D. H. Hyers and S. M. Ulam, Approximately convex functions, Proc. Amer. Math. Soc. 3 (1952), 821-828. MR 14:254b

[Ka] N. J. Kalton, The three space problem for locally bounded F-spaces, Compo. Math. 37 (1978), 243-276. MR 80j:46005

[KP] N. J. Kalton and N. T. Peck, Twisted sums of sequence spaces and the three space problem, Trans. Amer. Math. Soc. 255 (1979), 1-30. MR 82g:46021

[KPR] N. J. Kalton, N. T. Peck, and J. W. Roberts, An F-space sampler, Lecture Notes Series 89, London Mathematical Society, Cambridge University, 1984. MR 87c:46002

[KR] N. J. Kalton J. W. Roberts, Uniformly exhaustive submeasures and nearly additive set functions, Trans. Amer. Math. Soc. 278 (1983), 803-816. MR 85f:28006

[Ri] M. Ribe, Examples for the nonlocally convex three space problem, Proc. Amer. Math. Soc. 73 (1979), 351-355. MR 81a:46010

Departamento de Matemáticas, Universidad de Extremadura, Avenida de Elvas, 06071BADAJOZ, SPAIN

E-mail address: fcabello@unex.es 